\title{
Choroidal thickness and high myopia: a cross-sectional study and meta-analysis
}

\author{
Shiming Wang ${ }^{1 *}$, Yong Wang ${ }^{2}$, Xiaoming Gao ${ }^{1}$, Nana Qian ${ }^{1}$ and Youer Zhuo ${ }^{1}$
}

\begin{abstract}
Background: The purpose of this study was to examine the choroidal thickness of patients with high myopia using enhanced depth imaging optical coherence tomography (EDI-OCT) and compare them with healthy subjects.

Methods: We first conducted a cross-sectional study and then performed a meta-analysis to address this issue further. Using enhanced depth imaging optical coherence tomography (EDI-OCT), the macular choroidal thickness of high myopic eyes and normal control eyes were measured and compared at each location. Univariate and multivariate linear regression analyses were performed to assess the association between choroidal thickness and clinical factors such as axial length (AL), spherical equivalent (SE), and central corneal thickness. In the high myopic eyes, subgroup analysis of macular choroidal thickness was performed in eyes with or without lacquer cracks and choroidal neovascularization (CNV). The meta-analyses were conducted using the Stata software package.
\end{abstract}

Results: The high myopic eyes had a thinner choroid than the control eyes at all macular locations (all $P<0.001$ ). Multivariable linear regression analysis showed that the subfoveal choroidal thickness (SFCT) was not significantly thinner in association with the diagnosis. Subgroup analysis showed that the high myopia with CNV and with lacquer cracks had a significantly thinner choroid than without CNV or lacquer crack eyes. The result of our cross-sectional study is consistent with the results of the further meta-analysis with the pooled weighted mean difference (WMD) of $-116.30 \mu \mathrm{m}$ (95\% Cl: $-145.68,-86.92)$ for SFCT.

Conclusions: The present study, along with the comprehensive meta-analysis, indicated that in the Chinese population, the choroidal thickness in high myopic eyes was thinner than that of normal control eyes, even across different subgroups. This might be secondary to the longer AL but it is not an independent factor. The presence of CNV and of lacquer cracks is associated strongly with eyes with thinner macular choroids.

Keywords: Choroidal thickness, High myopia, Optical coherence tomography

\section{Background}

High myopia is one of the main causes of visual impairment worldwide [1]. It has been reported that about $1 \%$ of the population suffers from this disease [2]. High myopia is always accompanied by pathological structural changes, such as axial elongation, posterior scleral staphyloma, lacquer crack formation, thinning of the retina and chorioid, and choroidal neovascularization (CNV) [3-5]. Excessive axial elongation of the eyeball is thought to be one of the main causes of the ocular complications mentioned above. Among these complications, chorioretinal atrophy,

\footnotetext{
*Correspondence: wsm1981711@163.com

'Ningbo Aier Guangming Eye Hospital, 8 Huancheng West Road, 315020 Ningbo, China

Full list of author information is available at the end of the article
}

augmented by choroidal thinning, can lead to photoreceptor cell death, which results in the consequent loss of central visual function. Another notable complication threatening visual function is CNV [4]. Myopic CNV develops into secondary chorioretinal central atrophy and leads to central scotoma [6].

As we all know, in high myopic eyes, the earliest changes begin in the choroid [7]; recent interest has focused on the choroid as an important structure involved in the pathophysiology of high myopia [8]. Choroidal thickness may be an important parameter in studying the pathogenesis of high myopia. With the enhanced depth imaging (EDI) technique of optical coherence tomography (OCT) instruments, images of the choroid have improved, making it possible to measure choroidal

\section{Biomed Central}


thickness more accurately, safely, and simply [9-13]. Several researchers have found that in high myopic eyes, the choroidal thickness is significantly thinner than in normal eyes [14-17]. However, most of these studies failed to adjust for potential compounding factors such as axial length (AL) and spherical equivalent (SE), which have been proven to have a negative correlation with choroidal thickness $[18,19]$.

Therefore, the aim of this study was to evaluate the macular choroidal thickness in Chinese patients with high myopia and normal subjects and to determine the correlation with clinical factors in high myopic eyes. In addition, because of the myopic $\mathrm{CNV}$ and the lacquer crack, which would develop into secondary chorioretinal central atrophy, we compare the subfoveal choroidal thickness (SFCT) in high myopic eyes with or without $\mathrm{CNV}$ and with or without lacquer cracks. Finally, given the data accumulated, we sought to conduct a comprehensive meta-analysis to calculate the differences in SFCT between high myopic eyes and normal control eyes quantificationally.

\section{Methods}

\section{Subjects and enrollment criteria}

This study was approved by the Ethical Review Committee of Aier Eye Hospital and adhered to the provisions of the Declaration of Helsinki for research involving human subjects. Written informed consent was obtained from the participants before the study began. All the subjects were from a Chinese Han population, and they were prospectively and consecutively recruited for this study between October 2012 and November 2014.

In the high myopia group, the inclusion criteria were as follows: (1) high myopia was defined as an SE > -6 diopters (D); (2) all eyes had clear ocular media; (3) a clear image was obtained to enable precise measurement of the choroidal thickness. Patients were excluded from this study if they presented with any retinal abnormalities other than high myopia such as diabetic retinopathy, uveitis, drusen, retinal vascular abnormalities, age-related macular degeneration, or other eye diseases such as the history of amblyopia or glaucoma. Patients who had a history of intraocular surgery, refractive surgery, or intravitreal injection were also excluded from the study. Patients with systemic diseases were also excluded. In the control group, the inclusion criterion was healthy eyes with an SE between $-3 \mathrm{D}$ to $3 \mathrm{D}$.

\section{Examination}

All subjects underwent a refractive error examination without pupil dilation using autorefraction (NIDEK, AR-310A, Japan). SE was calculated as the sum of the spherical power and half of the cylinder power [20]. All the eyes of the subjects also underwent a thorough ophthalmic evaluation, including intraocular pressure
(IOP) measurement, slit-lamp biomicroscopy, dilated ophthalmoscopy fundus examination, and color fundus photographs (Canon, Retinal Camera CR-DGi, Japan). B-scanning was also performed. AL measurements were taken using partial optical coherence interferometry (IOLMaster; Carl Zeiss Meditec, La Jolla, CA, USA). All the repeat measurements used the median for the analysis. Demographic data on each subject's age and sex were collected.

\section{EDI-OCT examination}

All subjects were examined with a commercial OCT machine with an EDI mode (RS-3000 SD-OCT; Nidek, Japan). Choroidal imaging was performed as described in a previous study [10]. In brief, the vertical and horizontal sections going directly through the centre of the fovea were used for final analysis. Choroidal thickness was defined as the distance between the retinal pigment epithelium (outermost hyperreflective line) to the inner margin of the sclera. Choroidal thicknesses were measured at the subfovea, $1 \mathrm{~mm}$ and $3 \mathrm{~mm}$, nasally, temporally, superiorly, and inferiorly. All measurements were taken by a single experienced ophthalmologist who was masked to the clinical diagnosis of the subjects and was not involved in the data analysis. The images were obtained with the best visualization of the border between the choroid and the sclera known as the choroidal-scleral interface (CSI). If neither image had a clearly identifiable CSI, additional images were taken to produce the best possible view of the CSI. To avoid possible diurnal variation in choroidal thickness [21], all EDI-OCT examinations were performed between $9 \mathrm{AM}$ and $12 \mathrm{AM}$.

\section{Statistical analysis}

Statistical analysis was performed with SPSS software package version 17 (SPSS Inc, Chicago, IL, USA). Categorical covariates were assessed individually with the chisquare test. The demographics and ocular parameters between highly myopic and control eyes were compared using independent $t$-tests. One-way analysis of variance with the Bonferroni post-test was used to calculate the difference between the mean choroidal thicknesses of different locations. Univariate and multivariate regression analysis was used to evaluate the potential factors associated with SFCT in high myopia subjects. Univariate regression analyses were performed separately for each variable. Variables with a probability value $\leq 0.10$ in univariate analyses were included in the multivariate analysis using a stepwise method. In high myopic eyes, subgroup analysis of macular choroidal thickness was performed in eyes with or without lacquer cracks and CNV using an independent-sample $t$ test. For all the tests, $P<0.05$ was considered significant. 


\section{Meta-analysis}

Literature searches were performed in the following databases: PubMed, ISI Web of Science, EMBASE in English and in the Chinese National Knowledge Infrastructure (CNKI) (http://www.cnki.net/), VIP (http://www.cqvip. com/), and Wan Fang (http://www.wanfangdata.com.cn) in Chinese. The following search strategy was performed in PubMed: ((Choroidal[All Fields] AND thickness[All Fields]) OR ("choroid"[MeSH Terms] OR "choroid"[All Fields])) AND (("myopia"[MeSH Terms] OR "myopia"[All Fields]) OR ("myopia"[MeSH Terms] OR "myopia"[All Fields] OR "myopic"[All Fields])). The search results were supplemented by reviews of reference lists for all relevant studies and review articles. If there were several studies published by the same population, the recent study was included. The final literature search was updated December 2014, with no restrictions on publication year, language, or methodological filter.

Studies that met the following criteria were included in this meta-analysis: (a) cross-sectional or case-control design; (b) choroidal thickness measured by OCT; (c) differences in choroidal thickness between patients with high myopia and controls reported. The exclusion criteria were as follows: (a) duplicate data; (b) no control population; (c) abstracts, comments, letters, case report, reviews, or editorial articles; (d) insufficient data on choroidal thickness. Two observers independently extracted the following information from the included studies, using a standardized data extraction form: first author, year of publication, number of high myopia patients and controls, definition of high myopia, the SE criteria of control subjects, and differences in choroidal thickness. Any discrepancies were addressed by having a discussion to reach a consensus.

To arrive at a conservative estimate of the effect of potential population differences among the studies, we chose a random-effect model to calculate pooled weighted mean differences (WMDs) in the meta-analysis. Statistical heterogeneity among the studies was evaluated using Cochran's Q test and the $\mathrm{I}^{2}$ statistic. For the $\mathrm{Q}$ statistic, $P<0.05$ was considered to indicate statistically significant heterogeneity $[22,23]$. To explore the source of heterogeneity, subgroup analyses were done according to the instrument used, the mean age of included subjects, and the source of control. To evaluate the influence of an individual data set on the pooled results, one study was deleted at a time, and the combined estimates were recalculated based on the remaining studies. Potential publication bias was evaluated using funnel plots, Begg's test, and Egger's test $[24,25]$. A $P$ value less than 0.05 was considered statistically significant in the test results for overall effect. The meta-analyses were conducted using the Stata software package (Version 12.0; Stata Corp., College Station, TX).

\section{Results}

Demographic and baseline characteristics of the subjects A total of 314 eyes of 178 highly myopic patients and 173 eyes of 109 normal participants were included in the study. Of these, 13 high myopic eyes and eight control eyes were excluded because the border between the choroid and the sclera could not be visualized, although the optical media was clear. Therefore, data from 301 eyes of 171 high myopic patients and 165 eyes of 103 normal participants with high-quality OCT images were available and included in the analysis. The mean age was $22.23 \pm 6.50$ years in high myopia patients and $23.36 \pm$ 7.40 years in control subjects. The mean SEs for the high myopia group and the control group were $-7.56 \pm 1.99$ $\mathrm{D}$ and $-0.74 \pm 1.47 \mathrm{D}$, respectively. The mean ALs were $26.56 \pm 1.01 \mathrm{~mm}$ and $23.71 \pm 0.89 \mathrm{~mm}$ in the high myopia and control groups, respectively. The demographic and baseline characteristics of the patients are summarized in Table 1. There was a significant difference between the two groups in SE $(P<0.001)$ and AL $(P<0.001)$. The two groups did not differ significantly in terms of age, sex, IOP, central corneal thickness (CCT), diastolic blood pressure, systolic blood pressure, diastolic ocular perfusion pressure, systolic ocular perfusion pressure, or mean ocular perfusion pressure.

\section{Choroidal thickness in the macular region}

The choroidal thickness data at the fovea and $1 \mathrm{~mm}$ and $3 \mathrm{~mm}$ from the center temporally, nasally, superiorly, and inferiorly are shown in Table 2 . The high myopia group $(200.54 \pm 69.39 \mu \mathrm{m})$ exhibited a significantly thinner subfoveal choroid than the control group $(276.21 \pm 64.67 \mu \mathrm{m}$;

Table 1 Clinical characteristics in study subjects

\begin{tabular}{llll}
\hline & High myopia & Normal Control & $P$ \\
\hline No. of patients (No. of eyes) & $171(301)$ & $103(165)$ & - \\
Mean age (SD), y & $22.23(6.50)$ & $23.36(7.40)$ & 0.088 \\
Gender (male/female) & $119 / 52$ & $66 / 37$ & 0.345 \\
IOP at imaging (SD), mm Hg & $14.56(3.66)$ & $14.84(3.52)$ & 0.429 \\
Spherical equivalent (SD), D & $-7.56(1.99)$ & $-0.74(1.47)$ & $<0.001$ \\
Axial length (SD), mm & $26.56(1.01)$ & $23.71(0.89)$ & $<0.001$ \\
CCT, $\mu m$ & $544.61(33.23)$ & $541.44(33.63)$ & 0.328 \\
DBP, mmHg (SD) & $74.79(8.55)$ & $75.26(8.94)$ & 0.577 \\
SBP, mmHg (SD) & $120.29(11.65)$ & $122.53(13.81)$ & 0.064 \\
Diastolic OPP, mmHg (SD) & $60.23(9.04)$ & $60.42(9.62)$ & 0.829 \\
Systolic OPP, mmHg (SD) & $105.73(12.18)$ & $107.69(14.03)$ & 0.116 \\
Mean OPP, mmHg (SD) & $75.40(8.87)$ & $76.18(9.30)$ & 0.369 \\
\hline SD standsd
\end{tabular}

$S D$ standard deviation, $I O P$ intraocular pressure, $D$ diopter, $C C T$ central corneal thickness, DBP diastolic blood pressure, SBP systolic blood pressure, OPP ocular perfusion pressure

${ }^{1,2}$ Calculated as the differential pressure between diastolic or systolic blood pressure and IOP

${ }^{3}$ Calculated as the differential pressure between mean BP and IOP (mean $\left.\mathrm{BP}=\mathrm{DBP}+1 / 3^{*}(\mathrm{SBP}-\mathrm{DBP})\right)$ 
Table 2 Average choroidal thickness and $95 \% \mathrm{Cl}$ at different locations in macula

\begin{tabular}{|c|c|c|c|c|c|c|c|c|}
\hline \multirow{2}{*}{$\begin{array}{l}\text { Location } \\
\text { (mm from fovea) }\end{array}$} & \multicolumn{2}{|c|}{ High myopia } & \multicolumn{2}{|l|}{ Control } & \multirow{2}{*}{$\begin{array}{l}\text { Mean } \\
\text { Difference } \\
(\mu \mathrm{m})^{\mathrm{a}}\end{array}$} & \multicolumn{2}{|l|}{$95 \% \mathrm{Cl}(\mu \mathrm{m})$} & \multirow[t]{2}{*}{$P$} \\
\hline & Mean $(\mu \mathrm{m})$ & $\mathrm{SD}(\mu \mathrm{m})$ & Mean $(\mu \mathrm{m})$ & $\mathrm{SD}(\mu \mathrm{m})$ & & Lower Bound & Upper Bound & \\
\hline SFCT & 200.54 & 69.39 & 276.21 & 64.67 & -75.67 & -88.56 & -62.77 & $<0.001$ \\
\hline Superior 1 mm & 215.06 & 61.30 & 251.88 & 68.44 & -36.81 & -49.04 & -24.57 & $<0.001$ \\
\hline Superior 3 mm & 197.71 & 63.96 & 257.91 & 64.64 & -60.19 & -72.54 & -47.85 & $<0.001$ \\
\hline Inferior $1 \mathrm{~mm}$ & 198.90 & 61.66 & 242.11 & 73.16 & -43.21 & -56.51 & -29.91 & $<0.001$ \\
\hline Inferior $3 \mathrm{~mm}$ & 182.56 & 59.60 & 227.67 & 76.01 & -45.11 & -57.71 & -32.51 & $<0.001$ \\
\hline Nasal $1 \mathrm{~mm}$ & 171.76 & 66.11 & 226.07 & 76.26 & -54.30 & -67.60 & -41.00 & $<0.001$ \\
\hline Nasal 3 mm & 104.90 & 45.97 & 150.10 & 62.27 & -45.20 & -55.16 & -35.24 & $<0.001$ \\
\hline Temporal $1 \mathrm{~mm}$ & 189.29 & 67.02 & 250.52 & 69.04 & -61.22 & -74.12 & -48.33 & $<0.001$ \\
\hline Temporal 3 mm & 181.58 & 65.64 & 242.04 & 72.16 & -60.46 & -73.78 & -47.13 & $<0.001$ \\
\hline
\end{tabular}

Cl confidence interval, SD standard deviation, SFCT subfoveal choroidal thickness

${ }^{\text {a Normal group as reference }}$

$P<0.001)$. The macular choroidal thickness was significantly lower in the high myopia group than in the control group at all locations $(P<0.001)$.

\section{Univariate and multiple linear regression analysis}

Univariate and multivariate regression analyses were performed to determine the factors associated with SFCT in all subjects. Univariate regression analyses showed that diagnosis, SE, and AL factors were associated significantly with SFCT. In this univariate regression analyses, AL accounted for $31.8 \%$, SE explained $26.1 \%$, and diagnosis explained $22.1 \%$ of variation in choroid thickness. We then performed stepwise multiple linear analysis to determine the factors associated with SFCT in both groups. The model included the diagnosis, ES, and AL. After adjusting for the AL and ES by multivariate linear regression analysis, a diagnosis was not associated with SFCT $(P=0.471)$. Table 3 shows the detailed results of the linear regression analysis.

\section{Subgroup analysis of high myopia group}

To test whether patient with $\mathrm{CNV}$ or lacquer cracks in the high myopia group affected SFCT, the SFCT levels were compared between the patients with $\mathrm{CNV}$ and the patients without CNV. Table 4 summarizes the baseline characteristics of these two subgroups. The patients with CNV had a larger SE and longer AL than those without $\mathrm{CNV}$. There were no significant differences in other variables between the two subgroups. When we compared the SFCT between the two subgroups, we found that the high myopic eyes with CNV had a significant thinner choroid than high myopic eyes without CNV. We also divided the high myopic eyes into two subgroups based on their appearance of lacquer cracks. The baseline characteristics of the lacquer crack subgroup also had a larger SE and longer AL than the subgroup without lacquer cracks, and other variables were comparable between the two subgroups. When comparing the SFCT between the two subgroups, we also found that the high myopic eyes with lacquer cracks had a significantly thinner choroid than the high myopic eyes without lacquer cracks.

Table 3 Association between SFCT with other factors

\begin{tabular}{llll}
\hline Factor & Beta $(95 \% \mathrm{Cl})$ & Adjusted $\mathrm{R}^{2}$ & $P$ \\
\hline Univariate analysis & & & \\
$\begin{array}{l}\text { Diagnosis (high } \\
\text { myopia vs normal }\end{array}$ & $-75.67(-88.57,-62.77)$ & 0.221 & $<0.001$ \\
control) & & & \\
Age, year & $0.86(-0.17,1.88)$ & 0.004 & 0.100 \\
Gender (male/female) & $-6.85(-21.95,8.25)$ & 0 & 0.373 \\
IOP at imaging, & $-0.28(-2.22,1.67)$ & 0.002 & 0.781 \\
mm Hg & & & \\
Spherical equivalent, D & $10.52(8.91,12.13)$ & 0.261 & $<0.001$ \\
Axial length, mm & $-26.02(-29.48,-22.55)$ & 0.318 & $<0.001$ \\
CCT, $\mu m$ & $-0.16(-0.40,0.08)$ & 0.006 & 0.179 \\
DBP, mmHg & $0.66(-0.14,1.47)$ & 0.003 & 0.106 \\
SBP, mmHg & $0.41(-0.15,0.97)$ & 0.002 & 0.147 \\
Diastolic OPP, mmHg & $0.63(-0.13,1.38)$ & 0.004 & 0.104 \\
Systolic OPP, mmHg & $0.41(-0.13,0.95)$ & 0.003 & 0.138 \\
Mean OPP, mmHg & $0.72(-0.06,1.49)$ & 0.005 & 0.069 \\
Multivariate analysis & & & \\
Diagnosis (high & $-9.55(-35.57,16.48)$ & - & 0.471 \\
myopia vs normal & & & \\
control) & & - & 0.065 \\
Spherical equivalent $(\mathrm{D})$ & $3.36(-0.21,6.92)$ & - & \\
Axial length (mm) & $-21.93(-28.65,-15.22)$ & -001 \\
\hline SFCr subfovea choroda thckns, & &
\end{tabular}

SFCT subfoveal choroidal thickness, $\mathrm{Cl}$ confidence interval IOP intraocular pressure, $D$ diopter, $C C T$ central corneal thickness, $D B P$ diastolic blood pressure, SBP systolic blood pressure, OPP ocular Perfusion Pressure 
Table 4 Clinical characteristics in different high myopia subgroup

\begin{tabular}{|c|c|c|c|c|c|c|}
\hline \multirow[t]{2}{*}{ Variables } & \multicolumn{2}{|c|}{ With or without Lacquer Cracks subgroup } & \multirow[t]{2}{*}{$P$} & \multicolumn{2}{|c|}{ With or without CNV subgroup } & \multirow[t]{2}{*}{$P$} \\
\hline & Lacquer Cracks ( $n=32)$ & No Lacquer Cracks $(n=269)$ & & CNV $(n=21)$ & No CNV $(n=280)$ & \\
\hline Mean age (SD), y & $24.94(8.15)$ & $21.91(6.21)$ & 0.050 & $24.86(8.53)$ & $22.04(6.29)$ & 0.152 \\
\hline Gender (male/female) & $22 / 10$ & $189 / 80$ & 0.860 & $13 / 8$ & $198 / 82$ & 0.395 \\
\hline IOP at imaging (SD), $\mathrm{mm} \mathrm{Hg}$ & $15.63(3.51)$ & $14.43(3.65)$ & 0.077 & $15.10(3.80)$ & $14.52(3.65)$ & 0.487 \\
\hline Spherical equivalent (SD), D & $-10.25(2.63)$ & $-7.23(1.62)$ & $<0.001$ & $-10.66(2.10)$ & $-7.32(1.77)$ & $<0.001$ \\
\hline Axial length (SD), mm & $27.42(1.41)$ & $26.44(0.90)$ & $<0.001$ & $27.43(1.23)$ & $26.48(0.96)$ & $<0.001$ \\
\hline $\mathrm{CCT}, \mu \mathrm{m}$ & $554.15(37.42)$ & $543.47(32.58)$ & 0.131 & $546.38(41.53)$ & $544.47(32.61)$ & 0.800 \\
\hline $\mathrm{DBP}, \mathrm{mmHg}(\mathrm{SD})$ & $74.43(7.40)$ & $74.83(8.68)$ & 0.781 & $73.57(8.15)$ & $74.88(8.58)$ & 0.499 \\
\hline $\mathrm{SBP}, \mathrm{mmHg}(\mathrm{SD})$ & $119.87(11.47)$ & $120.33(11.68)$ & 0.832 & $118.57(8.55)$ & $120.41(11.84)$ & 0.485 \\
\hline Diastolic OPP, mmHg (SD) ${ }^{1}$ & $58.05(7.88)$ & $60.49(9.15)$ & 0.149 & $58.00(7.72)$ & $60.39(9.12)$ & 0.242 \\
\hline Systolic OPP, mmHg (SD) ${ }^{2}$ & $103.48(12.14)$ & $105.99(12.18)$ & 0.272 & $103.00(7.41)$ & $105.93(12.45)$ & 0.288 \\
\hline Mean OPP, mmHg (SD) $)^{3}$ & $73.19(7.69)$ & $75.65(8.97)$ & 0.138 & $72.99(6.44)$ & $75.58(9.01)$ & 0.199 \\
\hline SFCT & $145.03(73.53)$ & $207.14(65.97)$ & $<0.001$ & $144.14(54.54)$ & 204.77 (68.61) & $<0.001$ \\
\hline
\end{tabular}

CNV Choroidal neovascularization, SD standard deviation, IOP intraocular pressure, D Diopter, CCT central corneal thickness, DBP diastolic blood pressure, SBP systolic blood pressure, OPP ocular perfusion pressure, SFCT subfoveal choroidal thickness

${ }^{1,2}$ Calculated as the differential pressure between Diastolic or Systolic Blood Pressure and IOP

${ }^{3}$ Calculated as the differential pressure between mean BP and IOP (mean BP $=$ DBP $+1 / 3^{*}(\mathrm{SBP}-\mathrm{DBP})$ )

\section{Factors associated with SFCT in high myopic eyes}

Univariate and multivariate regression analyses were performed to determine the factors associated with SFCT in high myopic eyes. The variables associated significantly with SFCT by univariate regression were SE, AL, with $\mathrm{CNV}$, and with lacquer cracks. These associative variables were then entered into a multivariate regression analysis. Independent factors of choroidal thinning were $\mathrm{AL}$, the presence of $\mathrm{CNV}$, and the presence of lacquer cracks (all $P<0.05$ ). The results of these analyses are shown in Table 5.

\section{Eligible articles for meta-analysis}

To obtain more information about the difference in choroidal thickness between the highly myopic and normal control eyes, we performed a meta-analysis. The initial search yielded 891 potentially relevant studies. After the removal of duplicates found in the electronic databases, 627 studies remained. Based on their titles and abstracts, 609 articles were excluded because of their apparent irrelevance. Eighteen full-text articles were further assessed for eligibility. Finally, seven articles met the inclusion criteria and were included in this meta-analysis [14-17, 26-28]. Of these seven eligible articles, four were written in English [14-17], and three in Chinese [26-28]. The study selection process is shown in detail in Fig. 1.

\section{Characteristics of the included studies}

Overall, the seven studies plus the present study with 1204/630 high myopic eyes/control eyes were included in this meta-analysis. Among these studies, five originated from China, one from Spain, one from Singapore, and one from Japan. The used examination instrument included Heidelberg Spectralis, Topcon 3D-2000, Zeiss Cirrus, and Nidek RS-3000. In all included studies, the definition and the included high myopic eyes were the $\mathrm{SE}>-6 \mathrm{D}$. However, the SE of the control eyes included in different studies was not uniform and ranged from

Table 5 Association of factors with SFCT in highly myopic eyes

\begin{tabular}{lll}
\hline Factor & Beta $(95 \% \mathrm{Cl})$ & $P$ \\
\hline Univariate analysis & $0.40(-0.83,1.60)$ & 0.529 \\
Age, year & $-2.43(-19.64,14.79)$ & 0.782 \\
Gender (male/female) & $-1.29(-3.45,0.86)$ & 0.239 \\
IOP at imaging, mm Hg & $10.21(6.41,14.02)$ & $<0.001$ \\
Spherical equivalent, D & $-31.90(-38.86,-24.95)$ & $<0.001$ \\
Axial length, mm & $-0.16(-0.40,0.08)$ & 0.179 \\
CCT, um & $1.34(-0.43,2.25)$ & 0.112 \\
DBP, mmHg & $0.50(-0.18,1.18)$ & 0.147 \\
SBP, mmHg & $1.47(0.61,2.33)$ & 0.079 \\
Diastolic OPP, mmHg & $0.61(-0.04,1.25)$ & 0.064 \\
Systolic OPP, mmHg & $1.40(-0.53,2.28)$ & 0.067 \\
Mean OPP, mmHg & $-62.12(-86.70,-37.54)$ & $<0.001$ \\
Lacquer cracks (yes/no) & $-60.63(-90.80,-30.46)$ & $<0.001$ \\
CNV (yes/no) & & \\
Multivariate analysis & $-30.87(-38.15,-23.59)$ & $<0.001$ \\
Axial length, mm & $-28.76(-51.84,-5.67)$ & 0.015 \\
Lacquer Cracks (yes/no) & $-17.98(-33.25,-2.73)$ & 0.021 \\
CNV (yes/no) & $C 1$ confdenc &
\end{tabular}

SFCT subfoveal choroidal thickness, $\mathrm{Cl}$ confidence interval, IOP intraocular pressure, $D$ diopter, $C C T$ central corneal thickness, DBP diastolic blood pressure, SBP systolic blood pressure, OPP ocular Perfusion Pressure, CNV choroidal neovascularization 


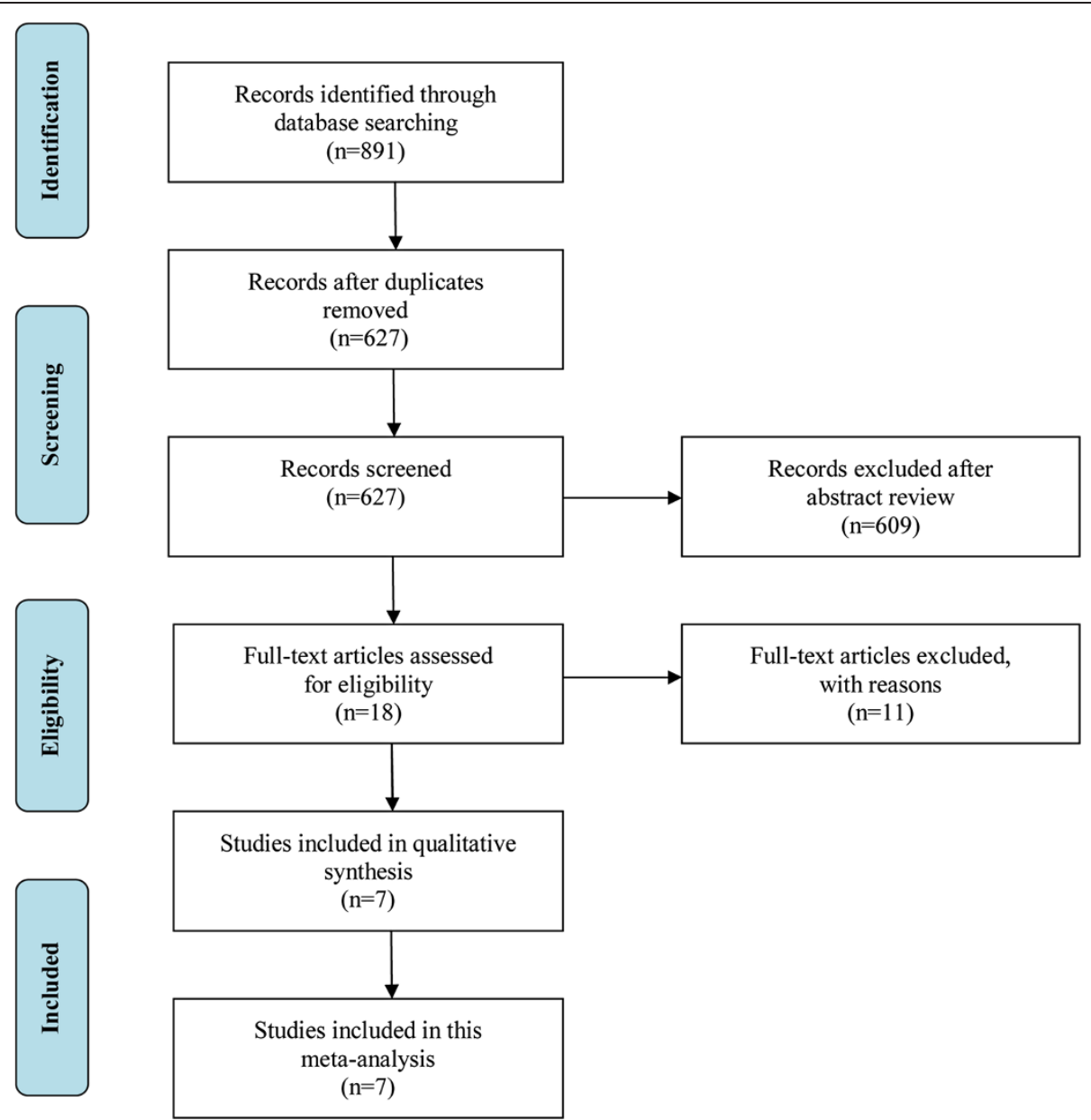

Fig. 1 Flow diagram outlining the selection process for inclusion of studies in the systematic review and meta-analysis

emmetropia to $\pm 6 \mathrm{D}$. The main characteristics of the included studies are presented in Table 6.

\section{Meta-analysis results}

In the comparison of the SFCT of high myopic eyes with the control eyes, the pooled WMDs were calculated using the random-effect model. The meta-analysis of these data showed that the SFCT was significantly thinner in the high myopic eyes than in the controls (WMD $=-116.30 \mu \mathrm{m}$; 95 \% CI: $-145.68,-86.92 ; P<0.001)$. Significant heterogeneity was observed between studies. Considering that the precision of the OCT was $10 \mu \mathrm{m}$, this difference could have been caused by instrument error. We then stratified the studies based on the instrument used. Subgroup analysis showed that a different examination instrument obtained similar results. In addition, the previous study had proved that choroidal thickness was negative association with age [19]. In the present meta-analysis, the mean age of included subjects in different studies ranged from 21.6 to 67.2 years, which might affect the pooled results. Therefore, we also stratified the studies according to the mean age of included subjects ( $>50$ years or $<50$ years). Both subgroups also showed a similar result in that the SFCT of high myopic eyes was significantly thinner than the control eyes. However, as expected, the $>50$ years subgroup showed the larger WMD than the $<50$ years subgroup. The detailed results are shown in Table 7 .

\section{Sensitivity analysis and publication bias}

To evaluate the influence of an individual data set on the pooled results, one study was deleted at a time. The corresponding estimates did not change greatly when any single study was deleted (Table 8).Publication bias was tested using Begg's test $(P=0.902)$ and Egger's test $(P=0.616)$, and no obvious evidence of publication bias was found.

\section{Discussion}

In this cross-sectional study, we measured the macular choroidal thickness in high myopic eyes and normal control eyes. The mean SFCT of the high myopia group in this study was $200.54 \pm 69.39 \mu \mathrm{m}$. Compared with the 
Table 6 Characteristics of included studies

\begin{tabular}{|c|c|c|c|c|c|c|c|}
\hline First Author (year) & Location & CT & Instrument & Age (year) & $\begin{array}{l}\text { Definition of } \\
\text { high myopia }\end{array}$ & $\begin{array}{l}\text { The SE criteria of } \\
\text { control subjects }\end{array}$ & $\begin{array}{l}\text { No eyes (High } \\
\text { myoipa/control) }\end{array}$ \\
\hline Flores-Moreno (2013) [16] & Spain & SFCT & Topcon 3D-2000 & $54.4 / 52.6$ & $\mathrm{SE}>-6.0 \mathrm{D}$ & $-6 \mathrm{D}$ to $+6 \mathrm{D}$ & $120 / 96$ \\
\hline Ohsugi (2013) [17] & Japan & SFCT & Heidelberg Spectralis & $65.4 / 67.2$ & $S E>-6.0 \mathrm{D}$ & $-3 \mathrm{D}$ to $+3 \mathrm{D}$ & $25 / 25$ \\
\hline Mo (2013) [28] & China & SFCT & Heidelberg Spectralis & $29.27 / 26.37$ & $\mathrm{SE}>-6.0 \mathrm{D}$ & Emmetropia & $96 / 72$ \\
\hline Gupta (2014) [14] & Singapore & SFCT & Heidelberg Spectralis & $21.59 / 22.06$ & $S E>-6.0 \mathrm{D}$ & $<0.5 \mathrm{D}$ & $520 / 128$ \\
\hline Chen (2014) [15] & China & SFCT & Zeiss Cirrus & $59.6 / 58.5$ & $S E>-6.0 \mathrm{D}$ & $-3 \mathrm{D}$ to $+3 \mathrm{D}$ & $36 / 42$ \\
\hline Qi (2014) [27] & China & SFCT & Heidelberg Spectralis & $33.51 / 34.70$ & $S E>-6.0 \mathrm{D}$ & $-2 \mathrm{D}$ to $+2 \mathrm{D}$ & $75 / 70$ \\
\hline Chen (2014) [15] & China & SFCT & Zeiss Cirrus & 29.1/29.6 & $S E>-6.0 \mathrm{D}$ & Emmetropia & $31 / 32$ \\
\hline Present (2014) & China & SFCT & Nidek RS-3000 & $22.23 / 23.36$ & $S E>-6.0 \mathrm{D}$ & $-3 \mathrm{D}$ to $+3 \mathrm{D}$ & $301 / 165$ \\
\hline
\end{tabular}

CT choroidal thickness, SE spherical equivalent, SFCT subfoveal choroidal thickness, D diopter

normal eyes, the results of this study revealed that the choroidal thickness was significantly smaller in the high myopic eyes. A thinning of the choroid in high myopic eyes has been demonstrated in several other studies [14-17]. More importantly, these findings are supported by the results of the present meta-analysis, which showed that the SFCT of high myopic eyes was significantly thinner than that of the normal control eyes with the pooled WMD of $-116.30 \mu \mathrm{m}$ (95\% CI: $-145.68,-86.92)$. This was not surprising. It has been reported that in high myopic eyes, excessive axial elongation of the eyeballs can cause biomechanical stretching and thinning of the choroid, retina, and sclera $[29,30]$. Thus, the thinner choroid is a long-term correlate of myopic axial elongation of the eye. In their previous studies, Gupta et al. [14] found that high myopic eyes have significantly thinner choroid than that of emmetropic eyes. However, they failed to adjust for relative compounding factors such as AL and SE, and we do not know whether the thinning choroid is because of the longer AL or an independent factor. Chen et al. [15] and Ohsugi et al. [17] both obtained results similar to those of Gupta et al. However, they also failed to adjust for any potential compounding factors that might affect the choroidal thickness such as age, AL, and SE. Thus, in the present study, we investigated whether the thinner choroid in high myopic eyes might be due to longer $\mathrm{AL}$ and larger SE. Surprisingly, after adjusting for the AL and SE by multivariate linear regression analysis, we found that the diagnosis was not associated with SFCT. Therefore, we concluded that the thinner SFCT in high myopic eyes might be secondary in its effects to the longer AL, but it is not an independent factor.

In this study, we also performed a meta-analysis to calculate the difference in the SFCT between high myopic eyes and normal control eyes quantificationally. After a systematic literature search of several databases, we reviewed seven studies, including ours, to compare the SFCT in high myopic eyes with that of normal control eyes. Our findings of significance were also reflected in the meta-analysis, even across different subgroups, which are less prone to chance results, indicating the robustness of our findings. It is also worth mentioning that the "leave-one-out" sensitivity analyses did not affect the pooled results of the metaanalysis, which illustrated that the present metaanalysis results were stable and reliable.

The formation of lacquer cracks is considered a risk factor for developing CNV [31, 32]. A previous study reported that the high myopic eyes with lacquer cracks

Table 7 Pooled estimates of all studies comparing SFCT in high myopic eyes with normal control eyes

\begin{tabular}{|c|c|c|c|c|}
\hline & No. of studies & WMD (random)(95 \% Cl) & Test for Heterogeneity & Test for Overall Effect \\
\hline \multicolumn{5}{|l|}{ Instrument } \\
\hline All trials & 8 & $-116.30(-145.68,-86.92)$ & $\mathrm{Q}=108.64, P<0.001$ & $\mathrm{Z}=7.76, P<0.001$ \\
\hline Heidelberg Spectralis & 4 & $-141.03(-159.43,-122.63)$ & $Q=75.46, P<0.001$ & $Z=15.02, P<0.001$ \\
\hline Zeiss Cirrus & 2 & $-80.36(-155.77,-4.95)$ & $Q=17.43, P<0.001$ & $\mathrm{Z}=2.09, P=0.037$ \\
\hline Topcon 3D-2000 & 1 & $-142.40(-167.45,-117.35)$ & - & $Z=11.14, P<0.001$ \\
\hline Nidek RS-3000 & 1 & $-75.67(-88.27,-63.07)$ & - & $Z=11.77, P<0.001$ \\
\hline \multicolumn{5}{|c|}{ The mean age of included subjects } \\
\hline All trials & 8 & $-116.30(-145.68,-86.92)$ & $\mathrm{Q}=108.64, P<0.001$ & $Z=7.76, P<0.001$ \\
\hline$>50$ years & 3 & $-128.19(-143.37,-113.01)$ & $\mathrm{Q}=1.98, P<0.001$ & $Z=16.55, P<0.001$ \\
\hline$<50$ years & 5 & $-109.56(-152.64,-66.47)$ & $\mathrm{Q}=101.70, P<0.001$ & $\mathrm{Z}=4.98, P<0.001$ \\
\hline
\end{tabular}

SFCT subfoveal choroidal thickness, WMD weighted mean differences, $\mathrm{Cl}$ confidence interval 
Table 8 Sensitivity analysis of the meta-analysis

\begin{tabular}{|c|c|c|c|c|c|}
\hline \multirow[b]{2}{*}{ Study Excluded } & \multicolumn{2}{|c|}{ Random effects model } & \multicolumn{3}{|c|}{ Test of homogeneity } \\
\hline & WMD & $95 \% \mathrm{Cl}$ & $\mathrm{Q}$ & $I^{2}(\%)$ & $P$ value \\
\hline None & -116.30 & $-145.68,-86.92$ & 108.64 & 93.6 & $<0.001$ \\
\hline Flores-Moreno (2013) [16] & -112.60 & $-145.01,-80.20$ & 102.87 & 94.2 & $<0.001$ \\
\hline Ohsugi (2013) [17] & -115.51 & $-148.03,-82.99$ & 108.29 & 94.5 & $<0.001$ \\
\hline Mo (2013) [28] & -115.95 & $-149.34,-82.56$ & 108.41 & 94.5 & $<0.001$ \\
\hline Gupta (2014) [14] & -111.37 & $-143.17,-79.56$ & 86.80 & 93.1 & $<0.001$ \\
\hline Chen (2014) [15] & -115.85 & $-148.61,-83.08$ & 108.43 & 94.5 & $<0.001$ \\
\hline Qi (2014) [27] & -109.84 & $-139.86,-79.82$ & 80.81 & 92.6 & $<0.001$ \\
\hline Chen (2014) [15] & -126.65 & $-154.96,-98.35$ & 79.35 & 92.4 & $<0.001$ \\
\hline Present (2014, unpublished) & -122.68 & $-150.54,-94.82$ & 59.02 & 89.8 & $<0.001$ \\
\hline
\end{tabular}

WMD weighted mean difference, $\mathrm{Cl}$ confidence Interval

had a less choroidal thickness than those without lacquer cracks [33]. El and associates [34] reported that macular choroidal thinning was also observed in high myopic eyes with CNV. In order to test whether the presence of $\mathrm{CNV}$ or lacquer cracks in high myopic eyes affected the SFCT, based on the relative large sample size, we divided the high myopic eyes into subgroups according to the presence or absence of CNV and lacquer cracks. The results showed that the subgroups with $\mathrm{CNV}$ and with lacquer cracks both had longer AL, larger $\mathrm{SE}$, and more importantly, a thinner choroid. In order to determine whether the thinner choroid is secondary to the longer AL or the larger SE, we performed the univariate and multivariate linear regression analysis again. In the univariable analysis, the results showed that $\mathrm{SE}, \mathrm{AL}$, the presence of lacquer cracks, and the presence of $\mathrm{CNV}$ were significantly associated with SFCT. After adjusting for the SE and AL by multivariate linear regression analysis, the mean SFCTs of the eyes with lacquer cracks and $\mathrm{CNV}$ were still significantly thinner than those where these were absent. This implies that the thinner choroid in the presence of $\mathrm{CNV}$ and the presence of lacquer cracks were not secondary to the longer AL or the larger SE but rather was an independent factor. This result might indirectly prove once again that myopic $\mathrm{CNV}$ and lacquer cracks could develop into secondary chorioretinal central atrophy. The SFCT could be a good indicator and predictor of the clinical significance and severity of the disease in these high myopic eyes.

Our results show that the average SFCT in high myopic eyes was $200.54 \pm 69.39 \mu \mathrm{m}$, which is much thicker than previous findings indicating a mean SFCT of 111.1 $\pm 45.0 \mu \mathrm{m}$ [15] and $115.0 \pm 85.3 \mu \mathrm{m}$ [16]. It was also significantly thicker than the pooled results of the present meta-analysis $(-116.30 \mu \mathrm{m})$. Considering this difference, we speculated that participants' ages might be the main cause. It has been proven that age is negatively associated with choroidal thickness. In our study, the mean age of high myopia is only 22.23 years, which is younger than in other studies [15-17]. Another important point is that, in all subjects, age was not associated with choroidal thickness, which is inconsistent with the findings of a large number of studies $[19,35,36]$. One possible explanation for this is that in our study, the age distribution of patients included is limited to a young age group; further studies including patients from a wide range of ages are needed to shed light on this difference. Another possible explanation is that other factors' strong association with the SFCT, such as AL and SE, concealed the factor of age. In the present study, we performed the EDI-OCT examination using the RS-3000 SD-OCT machine, which is different from the machines used in other studies. However, the results of the subgroup analysis showed that different examination instruments had similar results. This implied that using a different OCT machine would not affect the results. In addition, the previous studies proved that the choroidal thickness measurements obtained with different SD-OCTs were highly correlated and could be used interchangeably [37, 38].

Our study had a number of strengths. First, to date, the present study is the first synthesis exploring the association of choroidal thickness with high myopia. Second, the results of the present cross-sectional study were in accord with that of the corresponding meta-analysis. Third, our results are less prone to selection bias, in view of the low probability of publication bias.

It should be noted that the present study has a few limitations. First, in the meta-analysis, the SE of the control eyes included in the various studies was not uniform-it ranged from emmetropia to $\pm 6 \mathrm{D}$, which might affect the pooled results. Second, the small number of trials eligible for our meta-analysis made it difficult to acquire enough data to obtain meaningful results. Third, in the metaanalysis, substantial heterogeneity was observed among the studies. Although we performed analyses of the subgroups and sensitivity analyses, this heterogeneity 
could not be fully explained by the results. Fourth, the measurements of the choroidal thickness were performed manually, and automated software will be required for a more objective evaluation. However, the previous studies have proven that choroidal thickness measurement done using EDI-OCT is highly reproducible and repeatable [39, 40]. Finally, given that factors other than AL and SE likely affect the choroidal thickness, our conclusion should be viewed with caution.

\section{Conclusion}

The present study, along with the comprehensive metaanalysis, indicated that in the Chinese population, the choroidal thickness in high myopic eyes was thinner than that of normal control eyes, even across different subgroups. This might be secondary to the longer AL but it is not an independent factor. The presence of $\mathrm{CNV}$ and of lacquer cracks is associated strongly with eyes with thinner macular choroids. The SFCT might be a good indicator of the severity of myopic maculopathy.

\section{Competing interests}

The authors declare that they have no competing interests.

\section{Authors' contributions}

All authors conceived of and designed the experimental protocol. SW and YW collected the data. All authors were involved in the analysis. SW wrote the first draft of the manuscript. SW and XG reviewed and revised the manuscript and produced the final version. All authors read and approved the final manuscript.

\section{Acknowledgements}

This study was funded by Science Research Foundation of Aier Eye Hospital Group (Grant No.AF143D09)

\section{Author details}

${ }^{1}$ Ningbo Aier Guangming Eye Hospital, 8 Huancheng West Road, 315020

Ningbo, China. ${ }^{2}$ Wuhan Aier Eye Hospital, Wuhan, China.

Received: 8 January 2015 Accepted: 18 June 2015

Published online: 03 July 2015

\section{References}

1. Klaver CC, Wolfs RC, Vingerling JR, Hofman A, de Jong PT: Age-specific prevalence and causes of blindness and visual impairment in an older population: the Rotterdam Study. Arch Ophthalmol 1998, 116:653-658.

2. Vongphanit J, Mitchell P, Wang JJ. Prevalence and progression of myopic retinopathy in an older population. Ophthalmology. 2002;109:704-11.

3. Lim MC, Hoh ST, Foster PJ, Lim TH, Chew SJ, Seah SK, et al. Use of optical coherence tomography to assess variations in macular retinal thickness in myopia. Invest Ophthalmol Vis Sci. 2005;46:974-8.

4. Curtin BJ, Karlin DB. Axial length measurements and fundus changes of the myopic eye. Am J Ophthalmol. 1971;71:42-53.

5. Takano M, Kishi S. Foveal retinoschisis and retinal detachment in severely myopic eyes with posterior staphyloma. Am J Ophthalmol. 1999;128:472-6.

6. Yoshida T, Ohno-Matsui K, Yasuzumi K, Kojima A, Shimada N, Futagami S, et al. Myopic choroidal neovascularization: a 10-year follow-up. Ophthalmology. 2003;110:1297-305.

7. Saw SM, Gazzard G, Shih-Yen EC, Chua WH. Myopia and associated pathological complications. Ophthalmic Physiol Opt. 2005;25:381-91.

8. Ikuno Y, Tano Y. Retinal and choroidal biometry in highly myopic eyes with spectral-domain optical coherence tomography. Invest Ophthalmol Vis Sci. 2009;50:3876-80.
9. Hamzah F, Shinojima A, Mori R, Yuzawa M. Choroidal thickness measurement by enhanced depth imaging and swept-source optical coherence tomography in central serous chorioretinopathy. BMC Ophthalmol. 2014;14:145.

10. Zhou M, Wang W, Ding X, Huang W, Chen S, Laties AM, et al. Choroidal thickness in fellow eyes of patients with acute primary angle-closure measured by enhanced depth imaging spectral-domain optical coherence tomography. Invest Ophthalmol Vis Sci. 2013;54:1971-8.

11. Huang W, Wang W, Zhou M, Chen S, Gao X, Fan Q, et al. Peripapillary choroidal thickness in healthy Chinese subjects. BMC Ophthalmol. 2013;13:23.

12. Zhou M, Wang W, Huang W, Gao X, Li Z, Li X, et al. Is increased choroidal thickness association with primary angle closure? Acta Ophthalmol. 2014;92:e514-20.

13. Hirooka K, Tenkumo K, Fujiwara A, Baba T, Sato S, Shiraga F. Evaluation of peripapillary choroidal thickness in patients with normal-tension glaucoma. BMC Ophthalmol. 2012;12:29.

14. Gupta P, Saw S, Cheung CY, Girard MJ, Mari JM, Bhargava M, Tan C, Tan M, Yang A, Tey F, et al.: Choroidal thickness and high myopia: a case-control study of young Chinese men in Singapore. Acta Ophthalmol 2014. doi: 10.1111/aos.12631

15. Chen W, Song H, Xie S, Han Q, Tang X, Chu Y: Correlation of macular choroidal thickness with concentrations of aqueous vascular endothelial growth factor in high myopia. Curr Eye Res 2015, 40:307-313.

16. Flores-Moreno I, Lugo F, Duker JS, Ruiz-Moreno JM. The relationship between axial length and choroidal thickness in eyes with high myopia. Am J Ophthalmol. 2013;155:314-9.

17. Ohsugi $H$, Ikuno $Y$, Oshima K, Tabuchi H. 3-D choroidal thickness maps from EDI-OCT in highly myopic eyes. Optom Vis Sci. 2013;90:599-606.

18. Hirata M, Tsujikawa A, Matsumoto A, Hangai M, Ooto S, Yamashiro K, et al. Macular choroidal thickness and volume in normal subjects measured by swept-source optical coherence tomography. Invest Ophthalmol Vis Sci. 2011;52:4971-8.

19. Maul EA, Friedman DS, Chang DS, Boland MV, Ramulu PY, Jampel HD, et al. Choroidal thickness measured by spectral domain optical coherence tomography: factors affecting thickness in glaucoma patients. Ophthalmology. 2011;118:1571-9.

20. Gupta P, Cheung CY, Saw SM, Bhargava M, Tan CS, Tan M, et al. Peripapillary choroidal thickness in young asians with high myopia. Invest Ophthalmol Vis Sci. 2015;56:1475-81.

21. Tan CS, Ouyang Y, Ruiz H, Sadda SR. Diurnal variation of choroidal thickness in normal, healthy subjects measured by spectral domain optical coherence tomography. Invest Ophthalmol Vis Sci. 2012;53:261-6.

22. Li D, Zhou M, Peng $X$, Sun H. Homocysteine, methylenetetrahydrofolate reductase C677T polymorphism, and risk of retinal vein occlusion: an updated meta-analysis. BMC Ophthalmol. 2014;14:147.

23. Zhou M, Wang W, Huang W, Zhang X. Trabeculectomy with versus without releasable sutures for glaucoma: a meta-analysis of randomized controlled trials. BMC Ophthalmol. 2014;14:41.

24. Egger M, Davey SG, Schneider M, Minder C. Bias in meta-analysis detected by a simple, graphical test. BMJ. 1997;315:629-34.

25. Begg CB, Mazumdar M. Operating characteristics of a rank correlation test for publication bias. Biometrics. 1994;50:1088-101.

26. Chen L, Chen Z, Li Z. Subfoveal choroidal thickness measured by Cirrus HD optical coherence tomography in myopia. Int J ophthalmol. 2014;14:1641-3.

27. Qi H, Chen C, Weng M, Yi Z, Xu F. Change of choridal thickness and its influence factors in adult high myopic patients. Chin J Exp Ophthalmol. 2014;32:439-42.

28. Mo Y, Xiao X, He Y, Liu X, Xie L. Correlation between the thickness of different sites of retina and choroid in maculat area with central perimetry of high myopia. Chin J Pract Ophthalmol. 2013;31:128-30.

29. Wu PC, Chen YJ, Chen CH, Chen YH, Shin SJ, Yang HJ, Kuo HK: Assessment of macular retinal thickness and volume in normal eyes and highly myopic eyes with third-generation optical coherence tomography. Eye (Lond) 2008, 22:551-555.

30. Lam DS, Leung KS, Mohamed S, Chan WM, Palanivelu MS, Cheung CY, et al. Regional variations in the relationship between macular thickness measurements and myopia. Invest Ophthalmol Vis Sci. 2007;48:376-82.

31. Ikuno Y, Sayanagi K, Soga K, Sawa M, Gomi F, Tsujikawa M, et al. Lacquer crack formation and choroidal neovascularization in pathologic myopia. Retina. 2008;28:1124-31. 
32. Axer-Siegel R, Cotlear D, Priel E, Rosenblatt I, Snir M, Weinberger D, Indocyanine green angiography in high myopia. Ophthalmic Surg Lasers Imaging. 2004;35:139-45.

33. Wang NK, Lai CC, Chou CL, Chen YP, Chuang LH, Chao AN, et al. Choroidal thickness and biometric markers for the screening of lacquer cracks in patients with high myopia. PLoS One. 2013;8, e53660.

34. El ML, Bouladi M, Chebil A, Kort F, Largueche L, Mghaieth F. [Macular choroidal thickness assessment with SD-OCT in high myopia with or without choroidal neovascularization]. J Fr Ophtalmol. 2013;36:687-92.

35. Read SA, Collins MJ, Vincent SJ, Alonso-Caneiro D. Choroidal thickness in myopic and nonmyopic children assessed with enhanced depth imaging optical coherence tomography. Invest Ophthalmol Vis Sci. 2013;54:7578-86

36. Wang NK, Lai CC, Chu HY, Chen YP, Chen KJ, Wu WC, et al. Classification of early dry-type myopic maculopathy with macular choroidal thickness. Am J Ophthalmol. 2012;153:669-77.

37. Yamashita T, Yamashita T, Shirasawa M, Arimura N, Terasaki H, Sakamoto T. Repeatability and reproducibility of subfoveal choroidal thickness in normal eyes of Japanese using different SD-OCT devices. Invest Ophthalmol Vis Sci. 2012;53:1102-7.

38. Branchini L, Regatieri CV, Flores-Moreno I, Baumann B, Fujimoto JG, Duker $J S$. Reproducibility of choroidal thickness measurements across three spectral domain optical coherence tomography systems. Ophthalmology. 2012;119:119-23.

39. Shao L, Xu L, Chen CX, Yang LH, Du KF, Wang $S$, et al. Reproducibility of subfoveal choroidal thickness measurements with enhanced depth imaging by spectral-domain optical coherence tomography. Invest Ophthalmol Vis Sci. 2013;54:230-3.

40. Rahman W, Chen FK, Yeoh J, Patel P, Tufail A, Da CL. Repeatability of manual subfoveal choroidal thickness measurements in healthy subjects using the technique of enhanced depth imaging optical coherence tomography. Invest Ophthalmol Vis Sci. 2011;52:2267-71.

\section{Submit your next manuscript to BioMed Central and take full advantage of:}

- Convenient online submission

- Thorough peer review

- No space constraints or color figure charges

- Immediate publication on acceptance

- Inclusion in PubMed, CAS, Scopus and Google Scholar

- Research which is freely available for redistribution 